\title{
THE
}

9-15-1973

\section{Atomic and Nuclear Effects in the Slow-Neutron Total Cross Section of Terbium}

S. S. Malik

University of Rhode Island, smalik@uri.edu

M. Kamal

T. Turano

J. S. Desjardins

Follow this and additional works at: https://digitalcommons.uri.edu/phys_facpubs

Terms of Use

All rights reserved under copyright.

\section{Citation/Publisher Attribution}

Malik,S. S., Kamal, M., Turano, T., \& Desjardins, J. S. (1973). Atomic and Nuclear Effects in the SlowNeutron Total Cross Section of Terbium. Physical Review B, 8(6), 25952603. doi: 10.1103/

PhysRevB.8.2595

Available at: http://dx.doi.org/10.1103/PhysRevB.8.2595

This Article is brought to you for free and open access by the Physics at DigitalCommons@URI. It has been accepted for inclusion in Physics Faculty Publications by an authorized administrator of DigitalCommons@URI. For more information, please contact digitalcommons-group@uri.edu. 


\title{
Atomic and Nuclear Effects in the Slow-Neutron Total Cross Section of Terbium*
}

\author{
S. S. Malik, M. Kamal, ${ }^{\dagger}$ T. Turano, and J. S. Desjardins \\ Department of Physics, University of Rhode Island, Kingston, Rhode Island 02881 \\ (Received 31 May 1972; revised manuscript received 7 May 1973)
}

\begin{abstract}
The total cross section of terbium has been measured for neutron energies $E_{n}$ from 0.003 to $1.78 \mathrm{eV}$. For neutron energies above the Be cutoff a new method employing two crystal monochromators in series was used. For $E_{n} \lesssim 0.005 \mathrm{eV}$ a single-crystal monochromator in conjunction with a Be filter was employed. Using earlier measurements of the radiative-capture cross section, the experimental results have been analyzed to include the contributions due to paramagnetic scattering, coherent scattering, and phonon effects. The analysis shows that the experiment and the calculations are consistent everywhere except $0.015 \leqslant E_{n} \lesssim 0.10 \mathrm{eV}$. In this energy range inelastic coherent scattering is not accurately accounted for with the use of Placzek's incoherent approximations. In addition crystalline-field effects give rise to further complications. The comparison between the experiment and the calculations for $0.015 \lesssim E_{n} \lesssim 0.10 \mathrm{eV}$ suggests that studies of coherent inelastic scattering and crystalline-field effects in terbium metal are needed. The experimental results for $E_{n} \gtrsim 0.12 \mathrm{eV}$ yield the potential scattering cross section as $7.5 \pm 0.5 \mathrm{~b}$. This corresponds to a spin-independent nuclear radius of $7.73 \pm 0.27 \mathrm{fm}$ and to a radius parameter of $1.43 \pm 0.05 \mathrm{fm}$. The results suggest that the incoherent scattering cross section, if present, is very small $(\lesssim 1.0 \mathrm{~b})$.
\end{abstract}

\section{INTRODUCTION}

A large amount of data has been reported for slow-neutron total cross-section measurements. There is a qualified exception to this statement, namely, that the measurements relating to incident neutron energies $E_{n} \leqslant 0.05 \mathrm{eV}$ are fewer than those for $E_{n} \gtrsim 0.05 \mathrm{eV}$. This exception is even more evident for slow-ncutron cross sections of nuclei in the rare-earth region.

The purpose of this study was twofold. One was to make total cross-section measurements employing crystal monochromators covering the energy range from a few $\mathrm{eV}$ to a few $\mathrm{meV}$ in a continuous manner. The second objective is to attempt to explain the observed cross section in terms of partial cross sections. The predominant processes contributing to the total cross section for rare-earth nuclei in polycrystalline medium are radiative capture, coherent, incoherent, and magnetic scattering.

The monochromating system utilized a combination of two neutron monochromators in series. The crystalline form of the two monochromators to be used in such a system should have symmetry properties so that one of the monochromators yields a forbidden second-order reflection while the other yields a forbidden third-order reflection. The use of forbidden higher-order reflections dates back to Fermi ${ }^{1}$ and has been exploited in the form of a single-axis instrument either as a monochromator ${ }^{2-4}$ or as an analyzer. ${ }^{5}$ We know of no study where such a characteristic has been used to develop a two-axis neutron monochromator, although a twoaxis system for studying diffraction patterns has been studied by Caglioti and co-worker ${ }^{6,7}$ and by
Willis. ${ }^{8}$ A major result of these works is that intensity focusing results in the parallel combination of the two crystal monochromators.

We have successfully used the two-axis system to yield monochromatic neutron beams of high purity down to $E_{n}=0.014 \mathrm{eV}$. The low-energy limit was due to design constraints in the arm motions of the two axes. These constraints are now being partially removed. A study of the resolution-intensity characteristic of the two-axis system shows that in addition to focusing, the system is capable of superior resolution characteristics when compared to a single-axis monochromator employing similar collimators.

The total cross-section measurements were made by interposing the sample between the two monochromators. Such a procedure significantly reduces the effects of inelastic and multiple scattering. The reported values of the total cross section have an accuracy of $1 \%$ or better except for a few points in the neighborhood of $1 \mathrm{eV}$ where statistical errors are larger but always less than $5 \%$. The sample used was terbium metal. This particular choice was based on the fact that the element is a single isotope and that the independent measurements of radiative-capture cross section, ${ }^{9}$ paramagnetic form factor, ${ }^{10,11}$ and coherent-scattering cross section, ${ }^{11,12}$ all of good accuracy, were available.

Using the experimental results from the works mentioned above, present data have been subjected to a comprehensive analysis. The analysis includes generating contributions of various partial cross sections covering the range of energy pertinent to the present measurement. The paramagnetic cross section was obtained by numerically integrating the 
differential cross section employing measured values of the paramagnetic form factor. ${ }^{10}$ The coherent-scattering cross section included the inelastic contribution as well. This contribution was estimated using Placzek's incoherent approximation. ${ }^{13}$ The measured radiative-capture cross section ${ }^{9}$ was first analyzed in terms of contributions due to positive- and negative-energy resonances. These results were then utilized to generate the radiativecapture cross section at any desired value of energy.

The results of the analysis are that there is good agreement between the calculations and the measurements except in the energy range where Placzek's incoherent approximation for the estimation of inelastic coherent scattering is expected to be least applicable and where possible crystalline-field effects distort the paramagnetic-scattering cross section. The analysis of the data for $E_{n}>0.12 \mathrm{eV}$ (where results of experiment and analysis are in agreement) has been utilized to evaluate the potential-scattering cross section $\sigma_{p}$. The value of $\sigma_{p}$ resulting from the experiment and the analysis is $7.5 \pm 0.5 \mathrm{~b}$.

\section{A. Experimental Details}

The experimental arrangement employed is shown in Fig. 1. The neutron beam from the reactor was collimated using a $16^{\prime}$ collimator located in the beam pipe. The monochromator on the first axis of the two-axis system was $\mathrm{Be}(1011)$. The collimator after the first axis had a horizontal divergence of $12^{\prime}$. The sample mount was an $\mathrm{x}$-ray goniometer mounted on a slide which in turn was mounted on top of a 5-in. rotary table. The second axis followed the sample mount. The monochromator on the second axis was $\mathrm{Ge}(311)$ followed by an $18^{\prime}$ collimator. The neutron detector was a $\mathrm{He}^{3}$

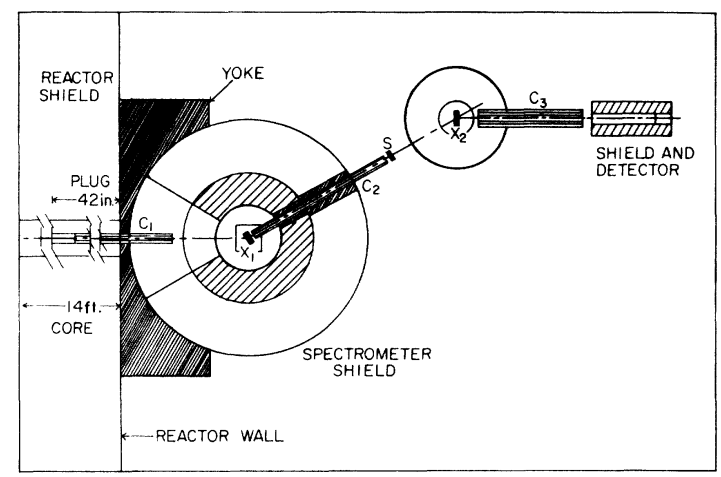

TWO AXIS NEUTRON SPECTROMETER

FIG. 1. Schematic diagram of the two-axis crystal monochromator. $C_{1}, C_{2}$, and $C_{3}$ are the Soller Collimators. $X_{1}$ and $X_{2}$ are the $\mathrm{Be}(10 \overline{1} 1)$ and $\mathrm{Ge}(311)$ crystal monochromators. $S$ is the sample. proportional counter. Reactor power was monitored using a $\mathrm{BF}_{3}$ proportional counter located vertically above the primary collimator at a distance of 27 in. The counter was directly exposed to the neutrons scattered by the collimator walls.

The sample was in the form of a disc of thickness $0.233 \mathrm{in}$. or 0.01854 atoms $/ \mathrm{b}$. The diffracted beam from the first axis was reduced to a circular cross section of $\simeq 1 \mathrm{~cm}^{2}$, while the sample cross section was $\simeq 4.5 \mathrm{~cm}^{2}$. The alignment of the sample with respect to the diffracted beam was accomplished by measuring the transmission of the beam for various angular settings of the sample on both sides of the beam line. The alignment so obtained showed that the sample was perpendicular to the beam within $2^{\circ}$, thereby introducing an error in the thickness of 6 parts in 10000 . The alignment error was thus of the same order as expected for the direct thickness measurements. The combined thickness-alignment error amounts to $0.1 \%$ of the value of the sample thickness.

The purity of the Tb sample according to the manufacturer was $99.9^{+} \%$. An activation analysis on the sample showed that maximum impurity was due to $\mathrm{Zr}(\simeq 500 \mathrm{ppm})$. The next lower concentration of impurity consisted of $\mathrm{Mn}$ and $\mathrm{Fe}$ (each $\simeq 200$ $\mathrm{ppm})$. The only traceable rare-earth impurity consisted of Dy $(\simeq 100 \mathrm{ppm})$.

The energy of the monochromatic neutron beam was determined by measuring the second-arm angle in both the parallel and the antiparallel positions. The absolute values of the two angles in the two arm positions agreed to within $5^{\prime}$ of arc. The angular separation $4 \theta$ in the two arm positions (parallel and antiparallel) was taken as the measure of the neutron energy.

The parallel and the antiparallel arrangement of the two axes, together with $2 \theta$ and $4 \theta$ are shown in Fig. 2. The maximum uncertainty in energy at $E_{n} \simeq 1.5 \mathrm{eV}$ for the Be (1011) monochromator was of the order of $0.01 \mathrm{eV}$. At $E_{n} \simeq 0.1 \mathrm{eV}$ for the same monochromator, the error corresponding to a maximum uncertainty of $5^{\prime}$ in $2 \theta$ amounts to less than $0.2 \%$.

B. Resolution Characteristics of Two-Axis System

The details of evaluating resolution characteristics of the double-crystal spectrometer, assuming that the crystals can be considered mosaic and that the transmission of the collimators can be approximated by Gaussians, are presented elsewhere. ${ }^{14}$ When both the crystals are set to pass the same nominal wavelength, the full width at halfmaximum of the intensity versus energy for the doubly diffracted monochromatic beam is given by

$$
\Delta E=\left(4 E_{0} \cot \theta_{0}\right)\left[4 p(\ln 2) /\left(4 p \gamma-\rho^{2}\right)\right]^{1 / 2},
$$

where $E_{0}$ is the nominal beam energy, $\theta_{0}$ is the 


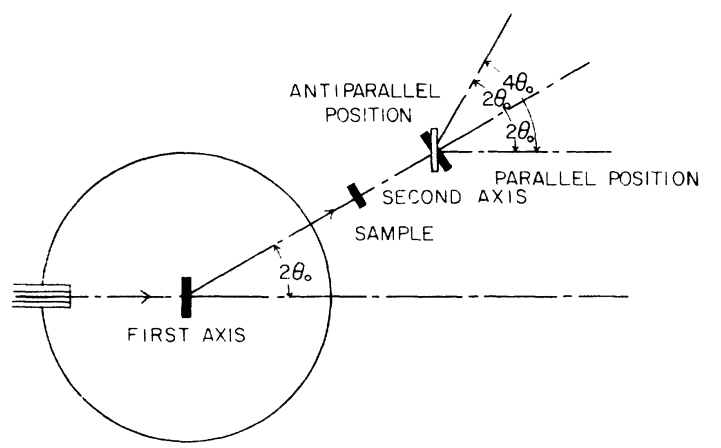

FIG. 2. Parallel and antiparallel arrangements of the two-axis system. $\theta_{0}$ is the Bragg angle (for the central ray) of the first monochromator. $\theta$ is the corresponding Bragg angle of the second monochromator.

Bragg angle of the first monochromator corresponding to the central ray, and

$$
\begin{aligned}
& \gamma \equiv 4(\ln 2)\left(\frac{1}{\beta_{1}^{2}}+\frac{4}{\alpha_{2}^{2}}+\frac{a^{2}+4+4 a \epsilon}{\beta_{2}^{2}}+\frac{4\left(1+a^{2}\right)+8 a \epsilon}{\alpha_{3}^{2}}\right), \\
& p \equiv 4(\ln 2)\left(\frac{1}{\alpha_{1}^{2}}+\frac{1}{\beta_{1}^{2}}+\frac{1}{\alpha_{2}^{2}}+\frac{1}{\beta_{2}^{2}}+\frac{1}{\alpha_{3}^{2}}\right) \\
& \rho \equiv 4(\ln 2)\left(\frac{2}{\beta_{1}^{2}}+\frac{4}{\alpha_{2}^{2}}+\frac{2 a \epsilon+4}{\beta_{2}^{2}}+\frac{4(1+a \epsilon)}{\alpha_{3}^{2}}\right) .
\end{aligned}
$$

Here $\alpha_{1}, \alpha_{2}$, and $\alpha_{3}$ are the full widths at half-maximum of the Gaussian angular transmission functions for collimators 1, 2, and 3 (see Fig. 1), $\beta_{1}$ and $\beta_{2}$ are the full width at half-maximum of the Gaussian mosaic plane distributions for crystals 1 and $2, a$ $\equiv\left(d \lambda / d \theta_{0}\right) /(d \lambda / d \theta)$ is the ratio of the dispersion in wavelength for crystals 1 and 2 , and $\epsilon=1$ for the antiparallel arrangement and -1 for parallel arrangement.

Table I shows three calculated values of $\Delta E$ for the present system at $E_{n}=0.015,0.15$, and $1.5 \mathrm{eV}$ and also includes the comparison with what would be expected with a single-axis instrument with the same set of the first two collimators.

\section{RESULTS}

The sample-out count $C_{0}$ is taken with both the crystal monochromators set to satisfy the Bragg condition. The sample is then remotely moved into the beam between the crystals and the count $C_{s}$ is taken. Background counting rates $B_{s}$ and $B_{0}$ with the sample in and out of the beam, respectively, are taken with the second monochromator set for Bragg reflection while the first monochromator is turned by $3^{\circ}$ away from the Bragg position. The background for sample in and out had the same value within the associated statistical uncertainties. Generally, the magnitude of the background was approximately $5 \%$ or less of the sample in, onBragg count.
Two specific sources of error in the cross-section measurement, namely, the sample thicknessalignment error and sample purity, have already been discussed. The other factors contributing to error considered here are (i) higher-order contamination, (ii) multiple and inelastic scattering, (iii) fluctuation in the monitor count rate due to reactor core adjustments and the changes in the room background (the statistical temporal fluctuations due to the fixed monitor counts are also a factor in contributing to the error), and (iv) statistical uncertainties in the signal.

A systematic search was made to detect second-, third-, and fourth-order contaminations in the doubly diffracted beam. Results showed that for the monochromator combination $\mathrm{Be}$ (1011) and $\mathrm{Ge}$ (311) all combined-order contaminations down to $E_{n}=14.5 \mathrm{meV}$ were less than $1 \%$. In addition, there was no indication of any Renninger effects where the neutron beam could, in principle, contain a different energy-intensity mixture. Since contaminations here are significantly below the background level, we have neglected any errors due to such causes.

In order to account for multiple and inelastic scattering contributions it is important to realize that the insertion of the sample between the two monochromators considerably reduces both these effects. The angular window set by the second monochromator rocking curve in the parallel arrangement is considerably narrower than the divergence of the beam incident upon the sample. In the present case the full width at half-maximum of the beam incident upon the sample was approximately $30^{\prime}$ of arc while the full width at half-maximum of the second monochromator rocking curve was $\approx 4^{\prime}$ of arc. The effect of multiple scattering on the observed transmission can be expressed as

$$
T_{\text {obs }}=T+\frac{d \Omega}{4 \pi} \frac{P_{x}\left(\sigma_{s} / \sigma\right)(1-T)}{1-\left(\sigma_{s} / \sigma\right)\left(1-P_{x}\right)} .
$$

In Eq. (2), $T_{\text {obs }}$ and $T$ are the observed and true transmission (in the absence of any multiple scattering). $\quad P_{x}$ is the probability that a neutron once scattered will escape the sample without further

TABLE I. Comparative Resolution Widths $\Delta E(\mathrm{eV})$ of single-axis and double-axis (parallel) arrangement using collimation half-angles and mosaic spreads of the experimental setup.

\begin{tabular}{cccc}
\hline \hline $\begin{array}{c}E_{n}(\mathrm{eV}) \\
\text { Type of Instrument }\end{array}$ & 0.015 & 0.15 & 1.5 \\
\hline Single Axis & 0.00009 & 0.0037 & 0.123 \\
$\begin{array}{c}\text { Double Axis } \\
\text { (parallel) }\end{array}$ & 0.00001 & 0.0017 & 0.055 \\
\hline \hline
\end{tabular}


interaction and represents an average probability independent of the neutron's past history. $\sigma_{s}$ and $\sigma$ are the scattering and the total cross sections, respectively.

The effects of multiple scattering in the observed values of the transmission for the present setup are small. To demonstrate this point we take the lowest-energy data point where $\sigma_{s} / \sigma \simeq 0.5$ and $T_{\text {obs }}$ $=0.122$. For the sample used, the escape probability using the calculations of Brunhart and Malik ${ }^{15}$ is $\simeq 0$. 18. For a sample to monochromator distance of $16 \mathrm{in.}$, a beam divergence of $10 \mathrm{mrad}$ and the monchromator height of 2 in., we find

$$
T_{\text {obs }}-T \simeq 0.13 d \Omega / 4 \pi \simeq 2 \times 10^{-4} \text {. }
$$

This leads to a correction of $0.1 \mathrm{~b}$ in the total cross section which is much smaller than the error due to statistical uncertainties.

The inelastic scattering contribution can be easily neglected because the inelastic scattering cross sections in the forward direction for the experimental energy-angle window amount to a small fraction of $1 \mathrm{mb}$ for a bound-atom scattering cross section of $20 \mathrm{~b}$.

The contribution due to the background fluctuations, reactor-core adjustments, and statistical temporal fluctuations can be estimated by measuring the distribution function of the signal-counter counting rates for fixed monitor counts. This procedure tests not only these three factors but the statistical reliability of the counting system as well. We had performed such a test on our system for a monitor count of 200000 (minimum count used in the present experiment). The results showed that the distribution function was normal. The standard deviation of the experimental distribution was $15 \%$ higher than would be expected if the monitor did not introduce additional uncertainties. Of the additional $15 \%$ increase in the standard deviation, approximately $7 \%$ arises due to the fixed monitor count of 200000 . The remainder of the increase in deviation is not abnormally high for effects of instrumental broadening. In calculating the errors in the cross section all standard deviations have been corrected for this effect.

We write for the transmission $T$ in the usual way,

$$
T=\left(C_{s}-B_{s}\right) /\left(C_{0}-B_{0}\right) \text {. }
$$

The cross section $\sigma$ and the statistical error $\Delta \sigma$ then are

$$
\sigma=(1 / n) \ln (1 / T)
$$

and

$$
\Delta \sigma=\frac{1}{n\left(C_{s}-B_{s}\right)}\left[\left(C_{s}+B_{s}\right)+T^{2}\left(C_{0}+B_{0}\right)\right]^{1 / 2} .
$$

Table II gives, as a function of neutron energy $E_{n}$,

\begin{tabular}{|c|c|c|c|c|}
\hline$E_{n}(\mathrm{eV})$ & $T$ & $\sigma(\mathrm{b})$ & $\Delta \sigma(\mathrm{b})$ & Monochromator Type \\
\hline 0.00354 & 0.122 & 113.3 & \pm 0.5 & $\mathrm{Be}$ \\
\hline 0.00364 & 0.128 & 110.9 & & filter \\
\hline 0.00375 & 0.126 & 111.7 & & plus \\
\hline 0.00387 & 0.127 & 111.3 & & $P b(111)$ \\
\hline 0.00400 & 0.135 & 108.0 & & monochromator \\
\hline 0.00414 & 0.137 & 107.2 & & \\
\hline 0.00429 & 0.138 & 106.8 & & \\
\hline 0.00446 & 0.149 & 102.7 & & \\
\hline 0.00456 & 0.154 & 100.9 & & \\
\hline 0.00480 & 0.159 & 99.2 & & \\
\hline 0.00500 & 0.162 & 98.2 & & \\
\hline 0.00520 & 0.165 & 97.2 & & \\
\hline 0.0145 & 0.301 & 64.8 & & $\operatorname{Be}(10 \overline{1} 1)$ \\
\hline 0.0154 & 0.307 & 63.7 & & plus \\
\hline 0.0163 & 0.318 & 62.0 & & $\mathrm{Ge}(311)$ \\
\hline 0.0173 & 0.324 & 60.8 & & monochromator \\
\hline 0.0186 & 0.343 & 57.7 & & \\
\hline 0.0205 & 0.344 & 57.6 & & \\
\hline 0.023 & 0.375 & 52.9 & & \\
\hline 0.024 & 0.379 & 52.3 & & \\
\hline 0.025 & 0.392 & 50.5 & & \\
\hline 0.026 & 0.394 & 50.2 & \pm 0.4 & $\mathrm{Be}(10 \overline{1} 1)$ \\
\hline 0.0275 & 0.416 & 47.3 & & plus \\
\hline 0.029 & 0.423 & 46.4 & & $\mathrm{Ge}(311)$ \\
\hline 0.031 & 0.437 & 44.7 & & monochromator \\
\hline 0.034 & 0.450 & 43.1 & & \\
\hline 0.036 & 0.460 & 41.9 & & \\
\hline 0.039 & 0.474 & 40.3 & & \\
\hline 0.042 & 0.488 & 38.7 & & \\
\hline 0.046 & 0.502 & 37.2 & & \\
\hline 0.050 & 0.518 & 35.5 & & \\
\hline 0.052 & 0.529 & 34.3 & & \\
\hline 0.054 & 0.538 & 33.4 & & \\
\hline 0.057 & 0.547 & 32.5 & & \\
\hline 0.060 & 0.555 & 31.8 & \pm 0.2 & \\
\hline 0.063 & 0.564 & 30.9 & & \\
\hline 0.066 & 0.571 & 30.2 & & \\
\hline 0.069 & 0.575 & 29.8 & & \\
\hline 0.073 & 0.597 & 27.8 & & \\
\hline 0.077 & 0.599 & 27.6 & & \\
\hline 0.084 & 0.618 & 26.0 & & \\
\hline 0.089 & 0.624 & 25.4 & & \\
\hline 0.095 & 0.633 & 24.7 & & \\
\hline 0.101 & 0.640 & 24.1 & & \\
\hline 0.108 & 0.650 & 23.2 & & \\
\hline 0.115 & 0.653 & 23.0 & & \\
\hline 0.124 & 0.665 & 22.0 & \pm 0.3 & $\operatorname{Be}(10 \overline{1} 1)$ \\
\hline 0.134 & 0.680 & 20.8 & & plus \\
\hline 0.144 & 0.689 & 20.1 & & $\mathrm{Ge}(311)$ \\
\hline 0.156 & 0.693 & 19.8 & & monochromator \\
\hline 0.170 & 0.705 & 18.9 & & \\
\hline 0.185 & 0.710 & 18.5 & & \\
\hline 0.203 & 0.719 & 17.8 & & \\
\hline 0.224 & 0.727 & 17.2 & & \\
\hline 0.247 & 0.735 & 16.6 & & \\
\hline 0.275 & 0.743 & 16.0 & & \\
\hline 0.308 & 0.749 & 15.6 & & \\
\hline 0.347 & 0.765 & 14.4 & & \\
\hline 0.394 & 0.765 & 14.4 & & \\
\hline 0.452 & 0.770 & 14.1 & & \\
\hline 0.523 & 0.797 & 12.2 & & \\
\hline 0.613 & 0.784 & 13.1 & & \\
\hline 0.728 & 0.812 & 11.2 & \pm 0.5 & \\
\hline 0.879 & 0.812 & 11.2 & & \\
\hline 1.082 & 0.821 & 10.6 & & \\
\hline 1.365 & 0.804 & 11.8 & & \\
\hline 1.780 & 0.758 & 14.9 & & \\
\hline
\end{tabular}
the values of $T$ and $\sigma$, monochromator combinations
TABLE II. Measured values of $T$ and $\sigma$ as a function of $E_{n}$. Included also are some typical values of $\Delta \sigma$.

and a few values of $\Delta \sigma$ to indicate the precision. The target thickness $n$ has been given earlier (0. 01854 atoms/b). 


\section{ANALYSIS}

Since terbium is expected to have significant contributions to the total cross section at low values of $E_{n}$ from resonances and paramagnetic scattering, we write

$$
\sigma_{T}=\sigma_{n, \gamma}+\sigma_{\mathrm{pm}}+\sigma_{c}+\sigma_{i}
$$

where $\sigma_{n, \gamma}$ is the radiative-capture cross section, $\sigma_{\mathrm{pm}}$ is the paramagnetic-scattering cross section, $\sigma_{c}$ is the coherent-scattering cross section, $\sigma_{i}$ is the incoherent-scattering cross section.

\section{A. Radiative-Capture Cross Section}

The radiative-capture cross section of $\mathrm{Tb}$ was recently measured using a Moxon-Rae detector at four neutron energies, ${ }^{9}$ namely, $E_{n}=0.033,0.075$, 0.1155 and $0.270 \mathrm{eV}$. These results are given in Table III.

In order to interpret the experimental values we fit an average $1 / v$ line through the four experimental values. The uncertainty associated with the $1 / v$ line in such a procedure is independent of energy. ${ }^{16}$ The fit to the experimental results corresponds to a value of $\sigma_{n, \gamma}$ given by

$$
\sigma_{n, \gamma}\left(E_{n}\right)=4.03 / E_{n}^{1 / 2} \pm 0.5 \mathrm{~b} \text {. }
$$

The neutron energy $E_{n}$ is in electron volts.

The assumption of $1 / v$ behavior for $\sigma_{n, \gamma}$ for the energies of Ref. 9 is well satisfied for the calculated contribution of all positive-energy resonances. The maximum departure occurs at $E_{n}$ $=0.270 \mathrm{eV}$ due to the $3.34-\mathrm{eV}$ resonance and amounts to $0.23 \mathrm{~b}$ if resonance parameters $\Gamma_{\gamma}$ $\simeq \Gamma=0.09 \mathrm{eV}$ and $\Gamma_{n}^{0}=0.0002 \mathrm{eV}$ are used.

The magnitude $\sigma_{n, \gamma}(E)=4.03 E_{n}^{1 / 2} \pm 0.5 \mathrm{~b}$ is much larger than the calculated contribution due to the positive-energy resonances. This contribution amounts to $\sigma_{n, \gamma}(E)=1.59 E^{-1 / 2} \mathrm{~b}$ over the energy range covered in the Moxon-Rae detector experiments. This value was obtained by using the recommended values of the resonance parameters up to $156 \mathrm{eV}$ whenever listed. In cases where no such values were available, average values of $2 g \Gamma_{n}^{0}$ and $\Gamma_{\gamma}$ were used. For resonances above $156 \mathrm{eV}$ the values of the $S$-wave strength function $S$, the reduced neutron width $\Gamma_{n}^{0}$, the radiative width $\Gamma_{r}$, and the average level spacing $D$ are needed. The values used for these parameters are $S=2.0 \times 10^{-4}$, $\Gamma_{n}^{0}=0.0002 \mathrm{eV}, \Gamma_{\gamma}=0.09 \mathrm{eV}$, and $D=5.4 \mathrm{eV}$.

The excess cross section at low energies was attributed to the presence of one or more negative-

TABLE III. Measured values of radiative-capture cross sections for the $\mathrm{Tb}$ element from Ref. 9 .

\begin{tabular}{lcccc}
\hline$E_{n}(\mathrm{eV})$ & 0.033 & 0.075 & 0.115 & 0.270 \\
$\sigma_{n, \gamma}(\mathrm{b})$ & $23.32 \pm 0.7$ & $\mathbf{1 5 . 6 1 \pm 0 . 5}$ & $11.32 \pm 0.5$ & $7.25 \pm 0.4$ \\
\hline
\end{tabular}

energy resonances.

Having obtained the contribution due to negativeenergy resonances, the values of $\sigma_{n, \gamma}$ can be calculated at all energies corresponding to the experimental data points. The only difference in these calculations was that the contribution of the 3.34$\mathrm{eV}$ level was separately calculated because it causes a departure from the $1 / v$ law as the neutron energy increases. Figure 3 exhibits the radiativecapture cross section together with the experimental values of the total cross section.

The procedure adopted here for the extrapolation of radiative-capture cross section into the energy regions not covered by the Moxon-Rae detector experiment leads to the following conclusions:

(i) Above $E_{n}=0.270 \mathrm{eV}$ the contribution of the 4. 99- and 11 . $14-\mathrm{eV}$ resonances is nearly $1 / v$ dependent even at $E_{n}=1.78 \mathrm{eV}$.

(ii) The uncertainties introduced by the extrapolation procedure are due mainly to the proximity of the 3.34-eV level. Here also only that part of $\sigma_{n, \gamma}$ which is in excess of the $1 / v$ dependent part, introduces extrapolation errors. The limits of the error estimated at higher-energy data points are illustrated by their values of, $\pm 0.35 \mathrm{~b}$ at $E_{n}$ $=1.78 \mathrm{eV} ; \pm 0.16 \mathrm{~b}$ at $E_{n}=1.08 \mathrm{eV}$ and $\pm 0.08 \mathrm{~b}$ at $E_{n}=0.613 \mathrm{eV}$.

\section{B. Paramagnetic Scattering}

The paramagnetic scattering cross section can be expressed in the usual form

$$
\frac{d \sigma_{\mathrm{pm}}}{d \Omega}=\frac{2}{3}\left(\frac{e^{2} \gamma^{2}}{2 m c^{2}}\right)^{2} \mu_{\text {eff }}^{2}|f(x)|^{2} e^{-2 W},
$$

where $e^{-2 W}$ is the Debye Waller factor. $2 W$ $=\left\langle u^{2}\right\rangle_{T} x^{2}$ where $\left\langle u^{2}\right\rangle_{T}$ is the mean-squared displacement of the atom at temperature $T$ (in $\left.{ }^{\circ} \mathrm{K}\right)$. $x$ is the momentum transfer $4 \pi \sin \theta / \lambda$ for the elastic scattering at an angle $2 \theta$ of a neutron of wavelength $\lambda . f(x)$ is the magnetic form factor corresponding to the momentum transfer $x . \mu_{\text {eff }}$ is the effective magnetic moment of the ion in the units of the Bohr magneton. $\gamma$ is the gyromagnetic ratio of neutron. $e^{2} / m c^{2}$ is the classical electron radius. Total paramagnetic scattering cross sections $\sigma_{\mathrm{pm}}\left(E_{n}\right)$ for experimental data points were obtained by numerically evaluating the integral

$$
\begin{aligned}
\sigma_{\mathrm{pm}}\left(E_{n}\right)=\left(6.26 \times 10^{-4}\right) \frac{\mu_{\text {eff }}^{2}}{E_{n}} & \int_{0}^{43.89 \sqrt{E_{n}}}|f(x)|^{2} \\
& \times\left[\mathrm{e}^{\left.-\left\langle u^{2}\right\rangle T^{x^{2}}\right] x d x .}\right.
\end{aligned}
$$

Equation (5) results from Eq. (4) if the integration variable is changed from the solid angle to the momentum transfer. In Eq. (5) $E_{n}$ is in electron volts and $\sigma_{\mathrm{pm}}$ is in barns.

A number of magnetization measurements ${ }^{17-19}$ of 


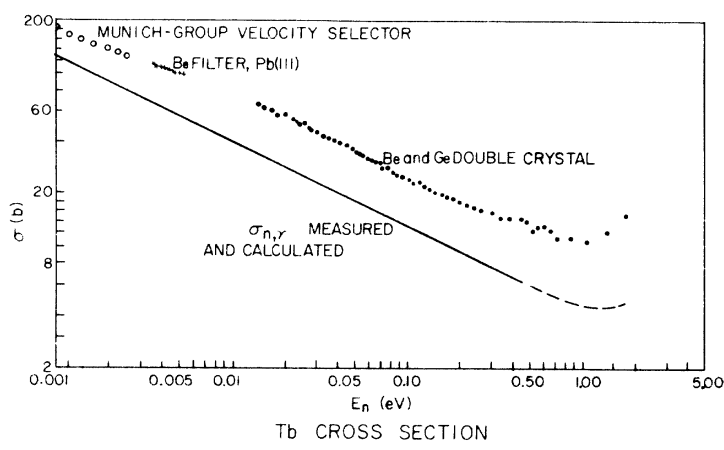

FIG. 3. Experimental values of the total cross section. Closed circles are data obtained using the twoaxis monochromator. Crosses are data obtained using $\mathrm{Be}$ filter and $\mathrm{Pb}(111)$ monochromator. Open circles are Munich group velocity selector data (BNL Report No. 325 , second edition, supplement No. 2, August, 1966). Solid curve represents the capture cross section calculated with the use of resonance parameters (BNL Report No. 325) and measured $\sigma_{n, \gamma}$ for $0.03<E_{n}<0.27$ eV Ref. 9.

terbium in the paramagnetic region have been made. The average of the results from these measurements yields a value of $\mu_{\text {eff }}=9.7 \mu_{B}$. Values of individual measurements lie within one percent of 9. $7 \mu_{B}$. The average value of $9.7 \mu_{B}$ compares very favorably with the theoretical prediction of $9.7 \mu_{B}$ calculated assuming a ${ }^{7} F_{6}$ state for the tripositive $\mathrm{Tb}$ ion. We have used the theoretical value in our calculations.

The value of $\left\langle u^{2}\right\rangle=0.01068 \AA^{2}$ at room temperature of $300^{\circ} \mathrm{K}$ was calculated in the Debye approximation using a Debye temperature of $160^{\circ} \mathrm{K} .{ }^{20}$

Accurate form-factor measurements for $\mathrm{Tb}$ using polarized and unpolarized neutrons have been made by Steinsvoll et al. ${ }^{21}$ and Brun and Lander. ${ }^{10}$ The results of Brun and Lander for $\sin (\theta) / \lambda \leq 0.15$ us ing the calculations of Lander and Brun ${ }^{22}$ were expressed as

$$
F(x)=0.86\left(\left\langle J_{0}\right\rangle+0.37037\left\langle J_{2}\right\rangle\right),
$$

where $\left\langle J_{0}\right\rangle$ and $\left\langle J_{2}\right\rangle$ are functions directly related to the radial wave functions of the $4 f$ electrons and have been tabulated by Blume et al. ${ }^{23}$

The values of $F(x)$ needed to evaluate $\sigma_{\mathrm{pm}}\left(E_{n}\right)$ from Eq. (5) were obtained as follows:

(a) For $0.15 \leq \sin (\theta) / \lambda \leq 1.3 \AA^{-1}, F(x)$ values were obtained from the tablulated values of Blume et $a l$, applying linear interpolation wherever necessary.

(b) For $\sin (\theta) / \lambda>1.3 \AA^{-1}, F(x)$ was approximated by the Gaussian $F(x)=e^{-0.01939 x^{2}}$.

This form of $F(x)$ was obtained by using the values of $F(x)$ for $x$ values in the neighborhood of $x$ $\simeq 1.3 \AA^{-1}$.

(c) For values of $\sin (\theta) / \lambda<0.15$, Eq. (6) was modified such that the factor 0.86 linearly approaches 1.0 at $\sin (\theta) / \lambda=0.05 \AA^{-1}$. Along with this modification linear interpolation was used to obtain the necessary $F(x)$ in the same manner as in (a).

The results of the computations of Eq. (5) are shown in Figure 4, together with the difference of the measured total cross section and the radiativecapture cross section extrapolated according to the procedure given in Sec. III A.

\section{CRYSTAL BINDING EFFECTS AND NUCLEAR SCATTERING}

\section{A. Coherent Elastic Scattering}

The coherent elastic scattering cross section for a polycrystal at neutron energy $E_{n}$ is given by

$$
\sigma_{c}^{\mathrm{e} 1}=\frac{0.041}{V E_{n}} b_{c}^{2} \sum_{\tau=\tau_{\min }}^{2 / \lambda} \eta_{\tau} F_{\tau} \frac{1}{\tau} e^{-Q \tau^{2}},
$$

where $\tau$ is the magnitude of a reciprocal lattice vector, $F_{\tau}$ is the structure factor for neutron scattering, $V$ is the volume of the unit cell, $b_{c}$ is the coherent scattering amplitude, $\eta_{\tau}$ is the multiplicity of reciprocal lattice vectors having magnitude $\tau$, and $Q=4 \pi^{2}\left\langle u^{2}\right\rangle_{T}$ and has a value of $0.422 \AA^{2}$. The structure factor $F_{\tau}$ for terbium is given by

$$
F_{\tau}=1+\cos \frac{1}{3} \pi\left(2 n_{1}+4 n_{2}+3 n_{3}\right) .
$$

The lattice constants are $a=3.599 \AA, c=5.696 \AA$. $\left(n_{1}, n_{2}, n_{3}\right)$ are the Miller indices of an arbitrary vector, $\vec{\tau}$, in the reciprocal lattice which is simple hexagonal. Since the point symmetry group of the simple hexagonal lattice contains 24 elements, the multiplicity $\eta_{\tau}$ is at most 24. Multiplicities of 12, 6 , and 2 also occur for $\vec{\tau}$ vectors oriented in special symmetry directions. $F_{\tau}$ is the same for all $\vec{\tau}$ vectors of a given magnitude. The value of $\left|b_{c}\right|$ used by us in evaluating $\sigma_{c}^{e 1}$ is that measured by Atoji, ${ }^{12}$ viz., 7.6 fm. Figure 5(a) shows our calculated value for this cross section.

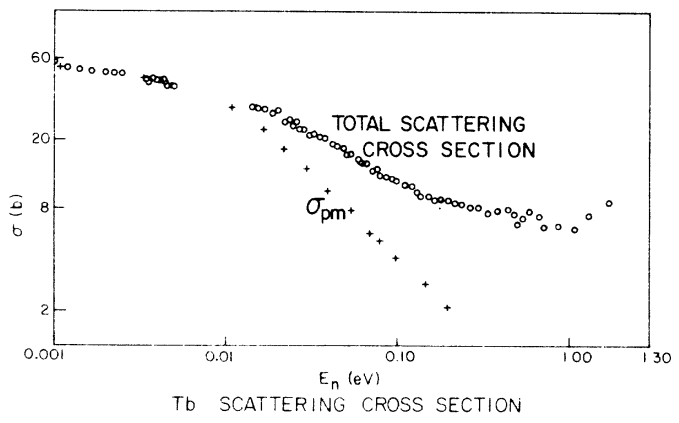

FIG. 4. Open circles are experimental values of the $\mathrm{Tb}$ total scattering cross section. Crosses are paramagnetic scattering cross section $\sigma_{\mathrm{pm}}$ calculated using Brun and Lander measured form factors Ref. 10. 

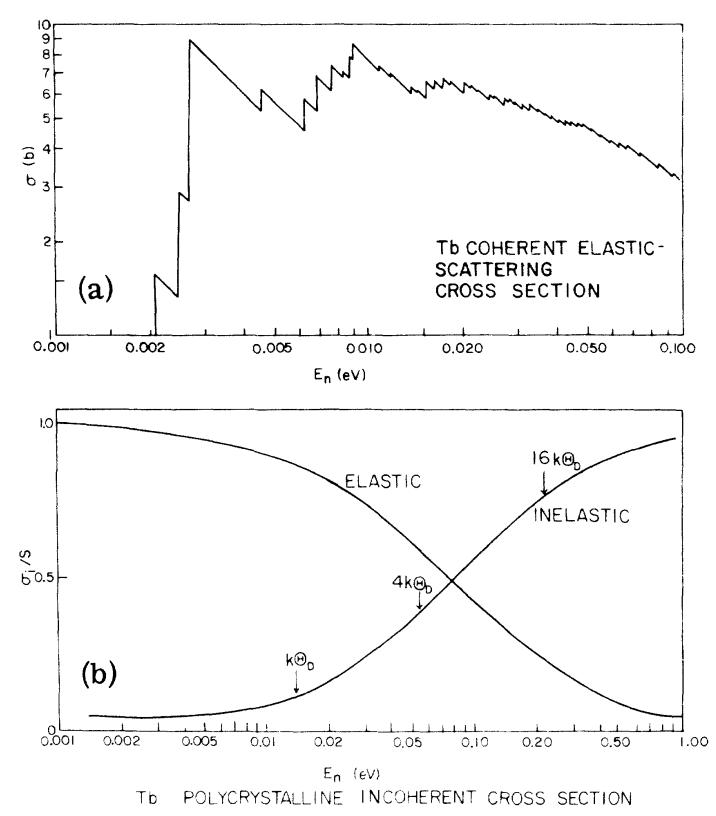

FIG. 5. (a) Tb coherent elastic scattering cross section calculated using $b_{c}=7.6 \mathrm{fm}$. The Debye-Waller factor has been assumed independent of the direction in the reciprocal lattice space. Debye temperature $\Theta_{D}$ was taken as $160^{\circ} \mathrm{K}$. (b) Incoherent elastic and inelastic scattering cross sections in the unit of $\sigma_{i}=4 \pi b_{i}^{2}$ calculated using Placzek's mass expansion and $\Theta_{D}=160^{\circ} \mathrm{K}$ and $M=159$. The inelastic contribution at $E_{n}=1,4$, and 16 times $\Theta_{D}$ is indicated.

\section{B. Coherent Inelastic Scattering Cross Section $\sigma_{c}^{\text {inel }}$}

Inelastic scattering here is referred to as the contribution to the cross section involving single and multiphonon processes. Direct evaluation of even the single phonon contribution to the coherent inelastic scattering presents a formidable computational problem. We have assumed that Placzek's incoherent approximation is applicable and that the interference effects are negligible.

The incoherent inelastic scattering cross section in terms of Placzek's expansion in the inverse power of mass $M$ (in the units of neutron mass) is ${ }^{13}$

$$
\sigma_{i}^{\text {inel }}=\left(\sum_{n=0}^{\infty} \sigma^{(n)} M^{-n}\right)-\sigma_{i}^{e 1}
$$

The expressions for $\sigma_{i}^{e 1}$ and $\sigma^{(n)}$ are given by Placzek. We have numerically evaluated $\sigma_{i}^{\text {e1 }}$ and $\sigma^{(n)}$. $\sigma_{c}^{\text {inel }}$ is then evaluated using the relation

$$
\sigma_{i}^{\mathrm{e}}=\sigma_{c}^{\mathrm{e}}\left(\sigma_{i}^{\mathrm{ine} 1} / \sigma_{i}^{\mathrm{e}}\right) \text {, }
$$

$\sigma_{i}^{e 1}$ and $\sigma_{i}^{\text {inel }}$ are plotted in Fig. 5(b).

\section{Potential Scattering}

The analysis of the data can be extended to incude evaluation of the potential scattering cross section $\sigma_{p}$. In the presence of resonances and other other partial cross sections one can write

$\sigma_{p}(E)=\left[\sigma(E)-\left(\sigma_{n, \gamma}(E)+\sigma_{\mathrm{pm}}(E)+\sum_{l}\left[\sigma_{I}^{l}(E)+\sigma_{\mathrm{res}}^{l}(E)\right]\right)\right]$,

where $\sigma_{I}^{l}(E)$ and $\sigma_{\text {res }}^{l}(E)$ are the interference and the resonance scattering cross sections due to the $l$ th resonance at neutron energy $E$.

Equation (8) suggests that the more accurate determination of $\sigma_{p}$ can be had by utilizing the $\sigma(E)$ data for the energy region where $\sigma_{n, \gamma}(E), \sigma_{\mathrm{pm}}(E)$, $\sum_{l} \sigma_{I}^{l}(E)$, and $\sum_{l} \sigma_{\text {res }}^{l}(E)$ are small. The data points corresponding to $E_{n} \gtrsim 0.12 \mathrm{eV}$ fit this criterion well. An additional reason to exclude data points below $E_{n} \simeq 0.12 \mathrm{eV}$ is due to the apparent uncertainty in the evaluation of the inelastic scattering cross section. This uncertainty is evident in the plot of $\sigma(E)-\left[\sigma_{n, \gamma}(E)+\sigma_{\mathrm{pm}}(E)\right]=\sigma_{b}$, as well as $\sigma_{b}$ $-\sigma_{c}$ shown in Fig. 6. The method of evaluating $\sigma_{n, \gamma}(E)$ and $\sigma_{\mathrm{pm}}(E)$ have been discussed earlier. $\sigma_{I}^{l}(E)$ and $\sigma_{\text {res }}^{l}(E)$ were evaluated using the resonance parameters listed in BNL Report No. 325. These contributions are significant only for the resonances at 3.34 and $11.14 \mathrm{eV}$. The contributions arising from resonances higher than $11.14 \mathrm{eV}$ and at negative energies were ignored. These two contributions tend to offset each other due to the change in the sign of the interference term.

The values of $\sigma_{p}(E)$ evaluated in accordance with Eq. (8) for all data points above $E_{n} \gtrsim 0.12 \mathrm{eV}$ has a small dispersion around an average value of $7.5 \mathrm{~b}$, except for a small structure around $E_{n}$

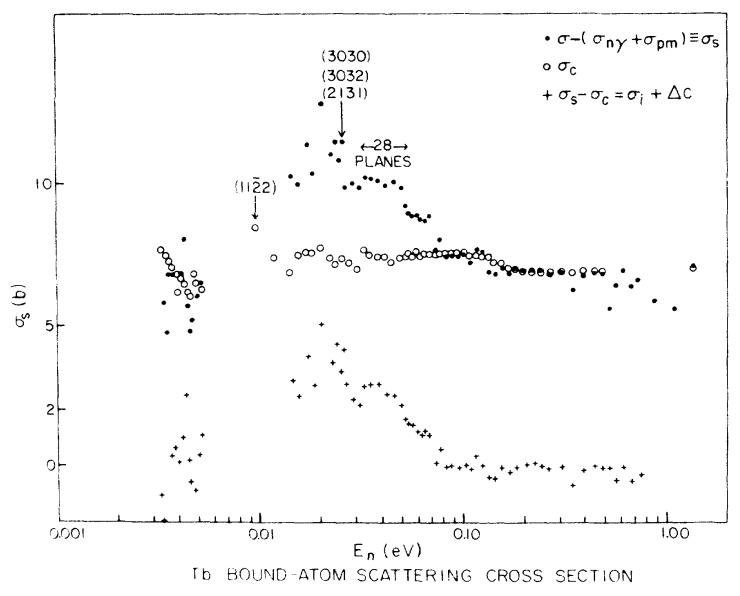

FIG. 6. Closed circles correspond to our values of the $\mathrm{Tb}$ bound-atom scattering cross section that remain after subtraction of the paramagnetic scattering cross section from the total scattering cross section. A few typical plane positions are indicated. The region $1 \leftarrow 28 \rightarrow 1$ has 28 diffracting planes. Open circles are some of the calculated values of $\sigma_{\mathrm{C}}$ (elastic plus inelastic in the incoherent approximation). Crosses show some typical values of $\left(\sigma_{S}-\sigma_{C}\right) . \Delta C$ is the extra cross section. 
$=0.6 \mathrm{eV}$. An Erbium impurity could introduce such a structure. However, the extent of the Erbium contamination required to explain the structure is much greater than the activation analysis suggests. Another possibility is that it may be a $p$-wave resonance.

A weighted average of the values of $\sigma_{p}$ using Eq. (8) yields a value of $\sigma_{p}=7.5 \pm 0.5 \mathrm{~b}$. The magnitude of the error is considered generous since it significantly exceeds the average dispersion of the $\sigma_{p}$ values at different energies. Assuming that the nuclear radius $R$ for the two spin states of the Tb nucleus is the same, one obtains $R=(1.43 \pm 0.05)$ $A^{1 / 3} \mathrm{fm}$. This value of nuclear radius for neutron interaction is slightly higher than the normally accepted value of $1.35 \mathrm{~A}^{1 / 3} \mathrm{fm}$. The slight increase in $R$ is, however, not unexpected since terbium lies in the mass-number region where an additional maximum in the $S$-wave strength function exists as a result of the spin-orbit interaction. ${ }^{24}$

\section{Inchoherent Scattering Cross Section}

If we disregard the anomalous behavior of $\sigma_{b}-\sigma_{c}$ in the energy region $0.015<E_{n}<0.10 \mathrm{eV}$, the rest of the data is consistent with zero or at most a value of about $1 \mathrm{~b}$ for the incoherent scattering cross section.

\section{CONCLUSION}

In this investigation the objective, as indicated in the Introduction, was to extend total cross-section measurements covering a wide range of neutron energies using a two-axis monochromator and then analyze the total cross section in terms of partial cross sections. This objective has been successfully accomplished. The results of the experiment and the analysis have been summarized in Fig. 7 where values of partial cross sections at $E_{n}=0.001 \mathrm{eV}$ are shown.

The energy range $0.01 \leqslant E_{n} \leqslant 0.10 \mathrm{eV}$ is known to be complicated, particularly for elements or isotopes having an appreciable coherent scattering cross section. It would seem desirable to study the coherent inelastic contributions and, if possible to correlate such contributions to the phonon dispersion relations which in most cases are experimentally accessible.

The rare-earth nuclei in solid solution make up rather complex magnetic scatterers. These nuclei, in general, have an appreciable portion of

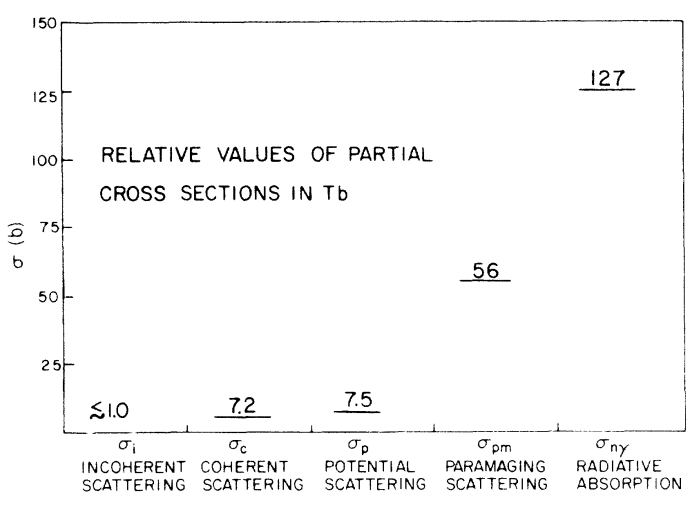

FIG. 7. Relative values of various $\mathrm{Tb}$ cross sections are shown with their numerical values. Values of $\sigma_{\mathrm{pm}}$ and $\sigma_{n, \gamma}$ have been rounded to the nearest whole number of barns and taken at $E_{n}=0.001 \mathrm{eV}$, the lowest energy considered in the analysis.

the atomic magnetic moment due to the orbital magnetic moments. Crystalline-field effects are also present ${ }^{25,26}$ but there is no clear cut evidence whether such effects contribute to an energy-dependent increase in the scattering cross section or, at $E_{n} \gtrsim 0.05 \mathrm{eV}$, begin to induce enough transitions among levels of different $m_{j}$ 's that partial or complete quenching of the orbital currents occurs. Scattering measurements at $E_{n} \simeq 0.1 \mathrm{eV}$ would be beneficial in attempting to answer such a question.

The value of the potential scattering cross section $\sigma_{p}$ of $7.5 \mathrm{~b}$ is substantially lower than the value $8.5 \mathrm{~b}$ listed in BNL Report No. 325. From the recent data on $\sigma_{p}$ values ${ }^{27}$ in the neighborhood of mass number $\mathrm{A}=160$, the value of $\sigma_{p}=7.5 \pm 0.5 \mathrm{~b}$ is quite reasonable.

A value of the incoherent scattering cross section $\sigma_{i} \leqslant 1 \mathrm{~b}$ suggests that for the $\mathrm{Tb}$ nucleus the two scattering lengths are essentially the same.

\section{ACKNOWLEDGMENTS}

The authors greatly appreciate the continued cooperation and help rendered by F. DiMeglio, director, and M. Doyle, assistant director of the Rhode Island Nuclear Science Center. The assistance of W. Magnan in the construction of the sample changer is also gratefully acknowledged. The authors are grateful to C. K. Unni for doing the activation analysis of the samples.
*Work partially supported by the U.S. Atomic Energy Commission under Contract No. AT(11-1)-3485.

'Present address: Instituto Venezolano de Investigaciones Cientificas, Caracas, Venezuela.

${ }^{1}$ E. Fermi and L. Marshall, Phys. Rev. 71, 666 (1947).

${ }^{2} J$. Hastings and L. Corliss (unpublished).

${ }^{3}$ J. M. Rustad, J. T. Wajima, and E. Melkonian, Bull. Am.
Phys. Soc. 4, 245 (1959).

${ }^{4}$ H. Bjerrum Moller, F. J. Shore, and V. L. Sailor, Rev. Sci. Instrum. 32, 654 (1961).

${ }^{5} \mathrm{G}$. Dolling and H. Nieman, Nucl. Instrum. Methods 49, 117 (1967).

${ }^{6} \mathrm{G}$. Caglioti, Acta Crystallogr. 17, 1202 (1964).

${ }^{7}$ G. Caglioti and F. P. Ricci, Nucl. Instrum. Methods 15, 155 
(1962).

${ }^{8}$ B. T. M. Willis, Acta Crystallogr. 13, 763 (1960).

${ }^{9}$ S. S. Malik, G. Brunhart, F. J. Shore, and V. L. Sailor, Nucl. Instrum. Methods 86, 83 (1970).

${ }^{10}$ T. O. Brun and G. H. Lander, J. Phys. (Paris) Colloq. 32, Cl-571 (1971).

${ }^{11}$ M. Atoji and D. E. Williams, J. Chem. Phys. 35, 1960 (1961).

${ }^{12}$ M. Atoji, J. Chem. Phys. 35, 1950 (1961).

${ }^{13}$ G. Placzek, Phys. Rev. 93, 895 (1954).

${ }^{14}$ S. S. Malik and J. S. Desjardins (unpublished).

${ }^{15}$ G. Brunhart and S. S. Malik, Bull. Am. Phys. Soc. 16, 15 (1971).

${ }^{16}$ Yardley Beers, Introduction to the Theory of Error

(Addison-Wesley, New York, 1957), p. 40.

${ }^{17}$ W. C. Thoburn, S. Legvold, and F. H. Spedding, Phys. Rev. 112, 56 (1958).

${ }^{18}$ S. Arajs and R. V. Colvin, J. Appl. Phys. 32, 3368 (1961).

${ }^{19}$ D. E. Hegland, S. Legvold, and F. Spedding, Phys. Rev.
131, 158 (1963).

${ }^{20}$ K. A. Gschneidner, Jr., in Solid State Physics, edited by F. Seitz and D. Turnbull (Academic, New York, 1964), Vol. 16, p. 371.

${ }^{21}$ O. Steinsvoll, G. Shirane, R. Nathans, M. Blume, H. A. Alperin, and S. J. Pickart, Phys. Rev. 161, 499 (1967).

${ }^{22}$ G. H. Lander and T. O. Brun, J. Chem. Phys. 53, 1387 (1970).

${ }^{23}$ M. Blume, A. J. Freeman, and R. E. Watson, J. Chem. Phys. 37, 1245 (1962).

${ }^{24}$ J. E. Lynn, The Theory of Neutron Resonance Reactions (Claredon, Oxford, England, 1968), p. 263.

${ }^{25}$ B. N. Brockhouse, L. N. Becka, K. R. Rao, R. N. Sinclair, and A. D. B. Woods, J. Phys. Soc. Jap. Suppl. 17, 63 (1962).

${ }^{26}$ T. O. Brun and G. H. Lander, Phys. Rev. Lett. 29, 1172 (1972).

${ }^{27}$ W. Dilg and H. K. Vonach, in Statistical Properties of Nuclei, edited by J. B. Garg (Plenum, New York, 1972), p. 332. 